eISSN: 2659-6482

DOI: https://doi.org/10.14201/pmrt.21644

\title{
UN VIAJE TURÍSTICO-MUSICAL POR ESPAÑA EN 1930. APROXIMACIÓN A LOS ACTOS DE BARCELONA Y VALENCIA
}

\section{A tourist-musical journey through Spain in 1930. Approach to the acts of Barcelona and Valencia}

\author{
Paula MOLINA GONZÁLEZ y Rebeca GEA MARTÍNEZ \\ Universidad de Salamanca \\ Máster en Música Hispana \\ paulamgcello@gmail.com \\ rebegeam@usal.es
}

RESUMEN: Kurt Schindler fue el principal creador de un viaje turístico-musical por España, realizado en el año 1930. Durante el desarrollo de esta iniciativa contó con el apoyo del Patronato Nacional de Turismo, el cual fue la plataforma principal para materializar la idea de este musicólogo alemán de nacionalidad estadounidense. En este artículo, nos proponemos describir tanto las redes personales como los eventos musicales de dicho viaje, centrándonos especialmente en las ciudades de Barcelona y Valencia. Para ello, nos vamos a servir principalmente de fuentes hemerográficas y documentales, así como de una bibliografía secundaria pertinente.

Palabras claves: siglo XX; folklore español; viaje turístico-musical; Kurt Schindler; Eduardo López-Chávarri; Francesc Pujol.

ABSTRACT: Kurt Schindler was the main creator of a tourist-musical journey through Spain in 1930. For this purpose, he had the support of the Patronato Nacional de Turismo, which was the main platform to materialize the idea of this German musicologist of American nationality. In this article, we propose to describe both the personal networks and the musical events of this journey, focusing especially on the cities of Barcelona and Valencia. 
PAULA MOLINA GONZÁLEZ Y REBECA GEA MARTÍNEZ

UN VIAJE TURÍSTICO-MUSICAL POR ESPAÑA EN 1930.

APROXIMACIÓN A LOS ACTOS DE BARCELONA Y VALENCIA

For this reason, we will mainly use from hemerographic and documentary sources, as well as a pertinent secondary bibliography.

Key words: Twentieth century; Spanish folklore; tourist-musical journey; Kurt Schindler; Eduardo López-Chávarri; Francesc Pujol.

\section{INTRODUCCIÓN}

En el período comprendido desde el 14 de mayo hasta el 9 de junio de 1930 se llevó a cabo un proyecto músico-cultural, el cual consistió en un tour por diferentes ciudades de la geografía española. En iniciativa de tal envergadura se vieron implicadas destacadas personalidades de la esfera sociopolítica y del panorama musical de la época. Dentro de este contexto, hay que destacar el papel del polifacético musicólogo alemán Kurt Schindler, referente tanto en la recopilación como en el estudio de la música tradicional y popular de nuestro país a principios del siglo XX.

El presente trabajo mostrará una aproximación a los principales protagonistas de este hecho histórico-musical, así como una descripción de las actividades del mismo. En concreto, nos centraremos en los eventos organizados en las ciudades del Levante: Barcelona y Valencia. Con este objetivo, hemos realizado un estudio crítico y comparativo de fuentes primarias de la época, entre las que se encuentran cartas y fuentes hemerográficas tanto nacionales como locales. De esta manera, se pueden entretejer las relaciones establecidas entre las diferentes personalidades que se citarán a continuación, esto es, las redes personales que nos ofrece el análisis de este tipo de fuentes.

\section{EL VIAJE TURÍSTICO-MUSICAL: UNA INICIATIVA DEL PATRONATO NACIONAL DE TURISMO}

La prensa española anunció entre el mes de abril y el mes de mayo de 1930 un viaje turístico-musical, como iniciativa del Patronato Nacional de Turismo. El anuncio se publicó a lo largo de esos dos meses en periódicos como La Revista de las Españas ${ }^{1}, A B C^{2}$, La Libertad ${ }^{3}$ y La Época (véase imagen 1). Estas fuentes

1. Sin firmar. (Abril 1930). Viaje turístico musical por España. Revista de las Españas, 44.

2. Sin firmar. (4 de mayo de 1930). ABC (Madrid), 38.

3. Sin firmar. (8 de mayo de 1930). Una bella iniciativa de arte. Excusión turístico-musical a través de España. La Libertad (Madrid), 8.

4. Sin firmar. (13 de mayo de 1930). Excursión turístico-musical por España. La Época, n. ${ }^{\circ} 28,1$. 
PAULA MOLINA GONZÁLEZ Y REBECA GEA MARTÍNEZ

UN VIAJE TURÍSTICO-MUSICAL POR ESPAÑA EN 1930.

APROXIMACIÓN A LOS ACTOS DE BARCELONA Y VALENCIA

detallan aspectos esenciales del viaje, como el recorrido, las actividades, las condiciones y el precio de los billetes, entre otros.

El objetivo de esta excursión, como se menciona en los anuncios, fue crear una experiencia que girara alrededor de la «música, y -casi no hay que decirlo- [de la] música popular española» ${ }^{5}$, así como «dar una síntesis completa de la variada riqueza musical y del folklore de España $»^{6}$. Además, fue una propuesta creada especialmente para «atraer (...) seguramente a gran número de extranjeros amantes de la música y a no pocos españoles, que difícilmente podrían gozar de una síntesis más afortunada del arte y la fisonomía popular de nuestro país» ${ }^{7}$.

Las ciudades elegidas para este propósito fueron Barcelona, Valencia, Granada, Sevilla, Madrid, Bilbao y San Sebastián. El recorrido empezó en Barcelona el 14 de mayo y siguió por el resto de las ciudades, en el orden citado, es decir, el viaje tuvo lugar en Valencia (durante los días 19 y 20 de mayo), Granada (del 22 al 24 de mayo), Sevilla (del 25 al 29 de mayo), Madrid (del 1 al 5 de junio), Bilbao (el 7 de junio) y, finalmente, San Sebastián (el 8 y el 9 de junio). Sin embargo, se incluyó una visita turística solamente a Córdoba, el día 30 de mayo, y otra a Toledo, durante el 2 y el 3 de junio.

Cada ciudad organizó una serie de actividades musicales. Para ello, desde el Patronato Nacional de Turismo se seleccionó a un elenco de músicos españoles como comité de dichas actividades. El comité estuvo constituido por compositores renombrados del momento, encargándose cada uno de ellos de la supervisión de una ciudad del viaje.

En primer lugar, para la supervisión de la zona de Levante, por un lado, se contó con Francesc Pujol para los eventos de Barcelona y, por otro, Eduardo López-Chávarri se encargó de las actividades de Valencia. En segundo lugar, Madrid estuvo bajo la supervisión de Enrique Fernández Arbós. En tercer lugar, la zona andaluza contó con la ayuda de Ángel Barrios para Granada y con el reverendo padre Eduardo Torres para Sevilla. Finalmente, las ciudades del País Vasco, Bilbao y San Sebastián, estuvieron bajo la revisión musical de Jesús Guridi y Antonio de Orueta, respectivamente.

5. Sin firmar. (8 de mayo de 1930). Una bella iniciativa de arte. Excusión turístico-musical a través de España. La Libertad (Madrid), 8; sin firmar. (4 de mayo de 1930). ABC (Madrid), 38.

6. Sin firmar. (13 de mayo de 1930). Excursión turístico-musical por España. La Época, n. ${ }^{\circ} 28,1$.

7. Sin firmar. (8 de mayo de 1930). Una bella iniciativa de arte. Excusión turístico-musical a través de España. La Libertad (Madrid), 8; sin firmar. (4 de mayo de 1930). ABC (Madrid), 38. 


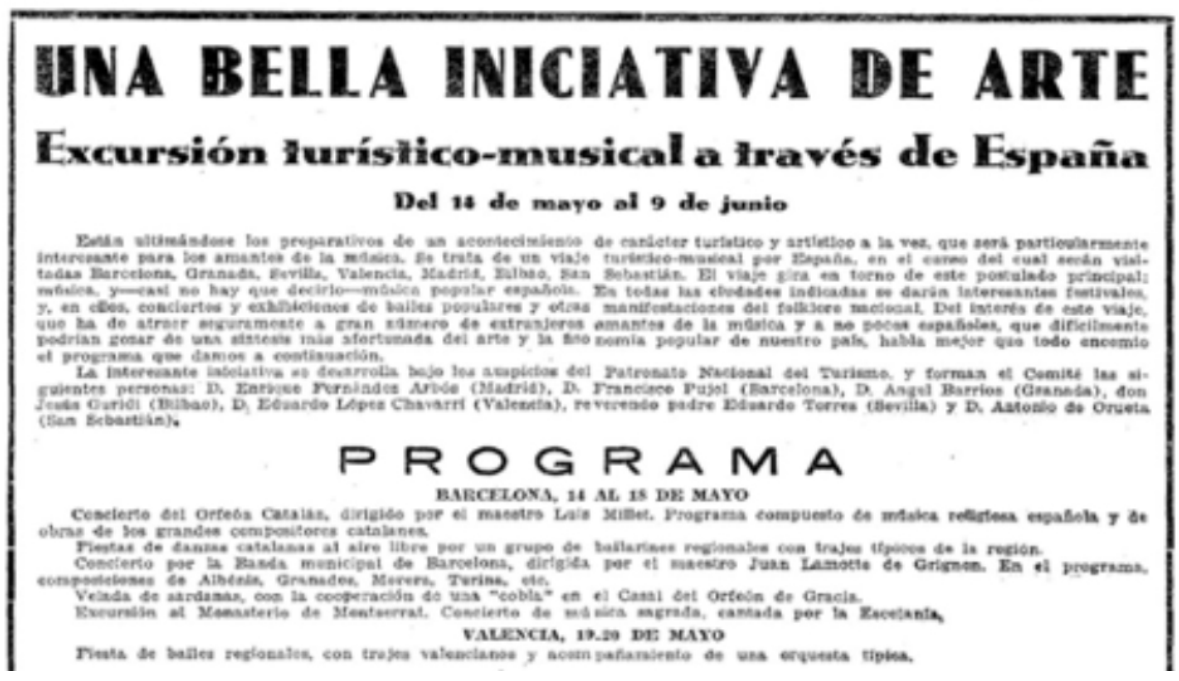

Imagen 1. Fragmento de prensa del anuncio del viaje en el periódico $A B C^{8}$.

De forma general, pasamos a detallar los eventos en cada una de estas ciudades, según el orden del programa del recorrido del viaje musical. Barcelona y Valencia se explicarán de forma más extensa en los apartados siguientes.

Las actividades en Granada se desarrollaron en dos acontecimientos. Por un lado, dos conciertos en los jardines de la Alhambra y el Albaicín, basados ambos en el cante «jondo» (seguidillas, polos, serranas, cañas, soleares, martinetes, carceleras, tonás y saetas viejas) y en cantos populares andaluces (rondeñas, malagueñas, granadinas, sevillanas, peteneras, saetas nuevas, cantos de cuna, villancicos, bailes sevillanos y fandanguillos). Por otro lado, se realizó un homenaje a Washington Irving en la Casa de los Tiros, con composiciones de Manuel de Falla, aunque no se especifican las obras musicales interpretadas en las fuentes consultadas.

Los eventos en Sevilla constaron de un concierto de música española de órgano a cargo de Norberto Almandoz, primer organista de la catedral, con un programa de la escuela clásica española, conformado por música de los siglos XVI y XVII, con composiciones de Antonio de Cabezón, Cristóbal de Morales y Sebastián Durón, entre otros. Además, se organizó también una sesión de bailes andaluces a cargo del maestro Otero.

La fiesta musical de Madrid constó de dos conciertos de la Orquesta Sinfónica bajo la dirección de Enrique Fernández Arbós. El programa estuvo basado en

8. Sin firmar. (4 de mayo de 1930). $A B C$ (Madrid), 38. 
PAULA MOLINA GONZÁLEZ Y REBECA GEA MARTÍNEZ

UN VIAJE TURÍSTICO-MUSICAL POR ESPAÑA EN 1930.

APROXIMACIÓN A LOS ACTOS DE BARCELONA Y VALENCIA

música de Isaac Albéniz, Enrique Granados, Joaquín Turina, Manuel de Falla, Óscar Esplá, Ernesto Halffter, Ruperto Chapí, Manuel Fernández Caballero, Gerónimo Giménez, F. A. Barbieri y Tomás Bretón, entre otros.

En Bilbao se organizó un programa coral con obras de Jesús Guridi a cargo de la Coral de Bilbao. Además, se interpretaron algunos bailes tradicionales como el ezpata-dantza o el aurresku. En San Sebastián actuó el Orfeón Donostiarra con una selección de autores guipuzcoanos, como Secundino Esnaola Berrondo, Pablo Sorozábal, José María Usandizaga y el padre José Antonio de San Sebastián, conocido este último como Padre Donostia o Aita Donostia.

Algunos de los anuncios de los periódicos ya citados también explicaban las condiciones y el precio del viaje. Se detallaba que los billetes incluían todos los gastos del recorrido, esto es, los «hoteles, transportes, coches-camas, "pullman", propinas e impuestos, entradas a las dos exposiciones, billetes para todas las funciones musicales, excursiones a Montserrat, Toledo y Córdoba»'. Los precios de los billetes variaban según las prestaciones ofertadas. Por ello, se vendieron tres tipos de billete: «clase gran lujo (3.350 ptas.), clase de lujo (2.500 ptas.) y primera clase $(1.900$ ptas. $) »^{10}$.

Sin embargo, se omite en estas fuentes al principal impulsor de esta iniciativa. Nos referimos a Kurt Schindler, el cual recorrió España y Portugal recopilando transcripciones y grabaciones de música popular en discos de aluminio. Estos documentos se publicaron póstumamente en 1941 con el nombre de Folk Music and Poetry of Spain and Portugal, constituyendo el cancionero más importante anterior a la Guerra Civil española. Estas investigaciones musicales en España estuvieron financiadas por el coleccionista americano Archer Milton Huntington, con un objetivo definido: hacer de la música popular española un patrimonio inmaterial de la Hispanic Society of America. Esta institución-museo recoge una completísima muestra de la cultura hispana en Nueva York. Así que no es de extrañar el interés tanto personal como económico de Schindler para que su empresa del viaje turístico-musical en España tuviera un éxito considerable.

A través de la correspondencia que mantuvo con Eduardo López-Chávarri ${ }^{11}$, se observa que la idea fue concebida por Kurt Schindler a principios de 1930. Posteriormente, utilizó el Patronato Nacional de Turismo como medio para desarrollar la iniciativa, convenciendo al delegado de esta institución en aquel momento, José Antonio de Sangróniz y Castro. Véase el siguiente extracto de

9. Sin firmar. (13 de mayo de 1930). Excursión turístico-musical por España. La Época, n. ${ }^{\circ} 28,1$; sin firmar. (8 de mayo de 1930). Una bella iniciativa de arte. Excusión turístico-musical a través de España. La Libertad (Madrid), 8.

10. Ídem.

11. Véase el apartado Epistolario. 
PAULA MOLINA GONZÁLEZ Y REBECA GEA MARTÍNEZ

UN VIAJE TURÍSTICO-MUSICAL POR ESPAÑA EN 1930.

APROXIMACIÓN A LOS ACTOS DE BARCELONA Y VALENCIA

la correspondencia entre Schindler y López-Chávarri: «Cuando propuse el plan de ese viaje musical al Secretario General José Antonio Sangróniz, estuvimos en fecha del 20 de Enero [de 1930]» ${ }^{12}$.

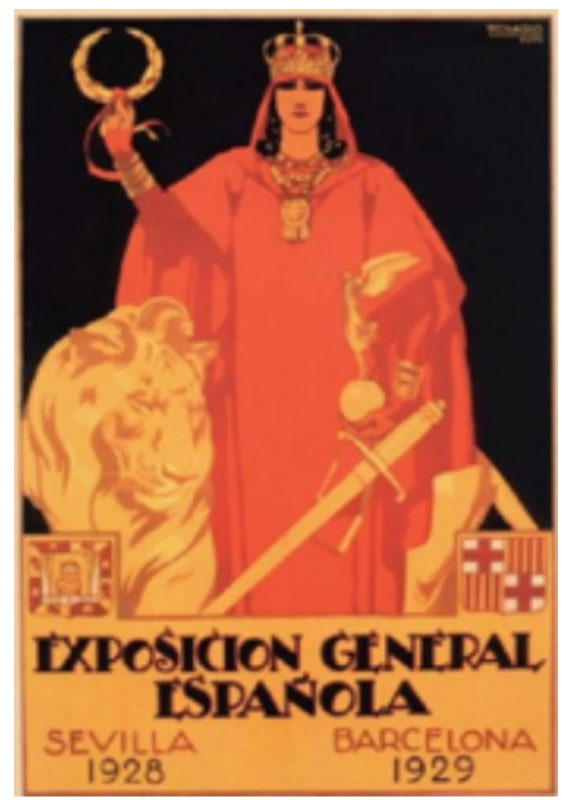

Imagen 2. Cartel de ambas exposiciones del dibujante y pintor Rafael de Penagos Zalabardo. Esta obra ganó el primer premio del concurso de carteles convocado para anunciar la Exposición General Española (Martín Emparan, 2008: 262).

Finalmente, hay que hacer una pequeña referencia al contexto del viaje, el cual no fue casualidad que se llevara a cabo en el año 1930, ya que coincidió con dos exposiciones importantes realizadas en España (véase imagen 2). Por esa razón, en la cita anterior en relación a los billetes se hace referencia a las dos exposiciones. Nos referimos, por un lado, a la Exposición Iberoamericana de Sevilla, la cual tuvo lugar entre el 9 de mayo de 1929 y el 21 de junio de 1930 con el objetivo de hermanar a los pueblos de España, Hispanoamérica, Estados Unidos, Portugal y Brasil. Por otro lado, la otra exposición tuvo lugar entre el 19 de mayo de 1929 y el 20 de enero de 1930, conocida como la Exposición Internacional de Barcelona.

12. Schindler, K. (9 de abril de 1930). [Carta para Eduardo López-Chávarri]. Biblioteca Valenciana Nicolau Primitiu. Fondo Familia Eduardo López-Chávarri Andújar. Signatura: AELCHE CR 2531.

Ediciones Universidad de Salamanca / 
PAULA MOLINA GONZÁLEZ Y REBECA GEA MARTÍNEZ

UN VIAJE TURÍSTICO-MUSICAL POR ESPAÑA EN 1930.

APROXIMACIÓN A LOS ACTOS DE BARCELONA Y VALENCIA

Sin embargo, las instalaciones siguieron abiertas al público hasta finales de 1930, de ahí que los viajeros musicales aún pudieron disfrutar de la exposición en la Ciudad Condal.

\section{BARCELONA}

El viaje turístico-musical por España comenzó el miércoles 14 de mayo de 1930, teniendo como primer destino Barcelona. En esta ciudad, se detuvieron durante cinco días, en los que se llevaron a cabo diferentes actividades relacionadas con la cultura catalana. Entre ellas, se realizaron conciertos de diversas agrupaciones musicales, una fiesta catalana, una velada de sardanas y una excursión.

Debemos resaltar que estos eventos, al igual que ocurrió en Sevilla, coincidieron con la presencia de la Exposición Internacional en Barcelona. Esta exposición se inauguró el 19 de mayo de 1929 con el propósito de proyectar la industria catalana en el exterior, dando a conocer los nuevos avances tecnológicos (Amiguet, 2015).

El recinto en el que se instaló dicha exposición fue construido siguiendo el proyecto del arquitecto Josep Puig i Cadafalch, en el cual se diferenciaban dos tipos de edificio. Por un lado, pabellones en los que se representaban distintos países como Francia, Bélgica, Alemania, Italia, Suiza, Dinamarca, Hungría, Noruega, Rumania, Japón o Estados Unidos. Por otro lado, palacios dedicados a las secciones oficiales del certamen.

En la organización de la jornada del viaje en Barcelona estuvo involucrado el musicólogo y compositor catalán Francesc Pujol, como miembro del comité constituido por los diferentes músicos que se encargaron de supervisar y representar a cada una de las ciudades implicadas. En el caso de Francesc Pujol, según la correspondencia entre Kurt Schindler y este, el musicólogo alemán le dio ciertas libertades en la elección de las fechas de los conciertos. En particular, Kurt Schindler trata con él lo referente al Orfeó Catala ${ }^{13}$, institución de la que Pujol formaba parte desde 1897 y la cual acabaría dirigiendo tras el fallecimiento de su maestro Lluís Millet.

En cuanto al itinerario del viaje en Barcelona consistió, como ya hemos mencionado, en el desarrollo de diversas actividades que se iniciaron tras la llegada de los turistas a la ciudad el día 14 de mayo.

El día siguiente, 15 de mayo, se realizó durante la mañana una visita a la Exposición Internacional. Después de ello, a las seis de la tarde tuvo lugar en la sala de espectáculos del Palacio de Proyecciones el primer concierto, a cargo de

13. Schindler, K. (3 de abril de 1930). [Carta para Francesc Pujol]. Biblioteca del Orfeó Català. Fondo Francesc Pujol. Signatura: CAT CEDOC 3.2 2367-2377. 
PAULA MOLINA GONZÁLEZ Y REBECA GEA MARTÍNEZ

UN VIAJE TURÍSTICO-MUSICAL POR ESPAÑA EN 1930.

APROXIMACIÓN A LOS ACTOS DE BARCELONA Y VALENCIA

la Banda Municipal de Barcelona. Esta fue una iniciativa del alcalde presidente de dicha Exposición Internacional, Juan Antonio Güell y López, en honor a los participantes en el evento turístico. La banda, que fue dirigida por el maestro Joan Lamote de Grignon, interpretó el siguiente programa dividido en dos partes y compuesto íntegramente por piezas de compositores españoles: Amadeo vives, Preludio de la ópera Maruxa; Enric Morera, Escena y Danza del Fuego de la ópera Tassarba; Joaquín Turina, Dos Danzas Fantásticas; Enrique Granados, Intermedio de Goyescas; Lamote de Grignon, Andalucía, cuadro sinfónico; Isaac Albéniz-Lamote de Grignon, Corpus Christi en Sevilla y El Albaicin (Suite Iberia); Lamote de Grignon (hijo), Nupcial (sardana); Gerónimo Jiménez, Intermedio de El Baile de Luis Alonso; José María Usandizaga, Pantomima de Las Golondrinas ${ }^{14}$.

Ese mismo día, a las diez de la noche hubo otro concierto de la Orquesta de Pau Casals en el Palacio de la Música Catalana. El aspecto más novedoso de este concierto fue el estreno en España de Manfred de Robert Schumann, basada en el poema homónimo de Lord Byron.

El 16 de mayo se programó una excursión al monasterio de Montserrat donde los monjes y la Escolanía ofrecieron un concierto de música sagrada, interpretándose la Salve Montserratina y el Virolay ${ }^{15}$.

El 17 de mayo continuaron las visitas, en este caso a los museos y monumentos más significativos de la ciudad. A las diez de la noche tuvo lugar en el Palacio de la Música Catalana un concierto del Orfeó Català, referente cultural del panorama musical catalán del siglo XX, bajo la batuta de su director, el maestro Lluís Millet. El programa que cantó dicha agrupación estuvo conformado por música religiosa del compositor catalán Antoni Nicolau (colaborador activo de esta formación coral), de Tomás Luis de Victoria y obras de compositores catalanes ${ }^{16}$, entre los que se encontraban discípulos del propio Lluís Millet ${ }^{17}$.

El último día en Barcelona, 18 de mayo, se volvió a visitar la Exposición Internacional. Después de ello, a las seis de la tarde se desplazaron hacia la Plaza Mayor del Pueblo Español, donde se había preparado una fiesta de danzas catalanas. En dicha fiesta se representó una «boda montañesa», como propuesta de Francesc Pujol, en la que intervinieron bailarines y músicos con trajes e instrumentos propios de la región ${ }^{18}$. La jornada finalizó con una velada de sardanas a las diez de la noche, la cual se celebró también en honor a los adheridos al viaje turístico.

14. Sin firmar. (14 de mayo de 1930). Palacio de Proyecciones. La Vanguardia, 22.

15. Sin firmar. (13 de mayo de 1930). Excursión turístico-musical por España. La Época, n. ${ }^{\circ} 28,1$.

16. Ídem

17. Sin firmar. (Junio de 1930). Orfeó Català. Música, n. ${ }^{\circ}$ 10, 20.

18. Sin firmar. (4 de mayo de 1930). $A B C$ (Madrid), 38. 
PAULA MOLINA GONZÁLEZ Y REBECA GEA MARTÍNEZ

UN VIAJE TURÍSTICO-MUSICAL POR ESPAÑA EN 1930.

APROXIMACIÓN A LOS ACTOS DE BARCELONA Y VALENCIA

Esta se realizó en la Casa del Orfeó Gracienc, dirigida por el maestro Joan Balcells y con la colaboración de una agrupación instrumental típica de Cataluña, conocida como «cobla». En el repertorio se interpretaron algunas canciones populares entre las que estaba Kalinka, armonizada por Kurt Schindler, quien había trabajado en este proyecto musical y formaba parte de los excursionistas. Otra de las obras a resaltar fue La sardana de les monges de Enric Morera, la cual se interpretó dos veces, la segunda dirigida por el propio compositor, quien también estaba entre los presentes. La velada terminó con La Font de l'Albera, obra igualmente de Morera en la que colaboró la «cobla» ${ }^{19}$.

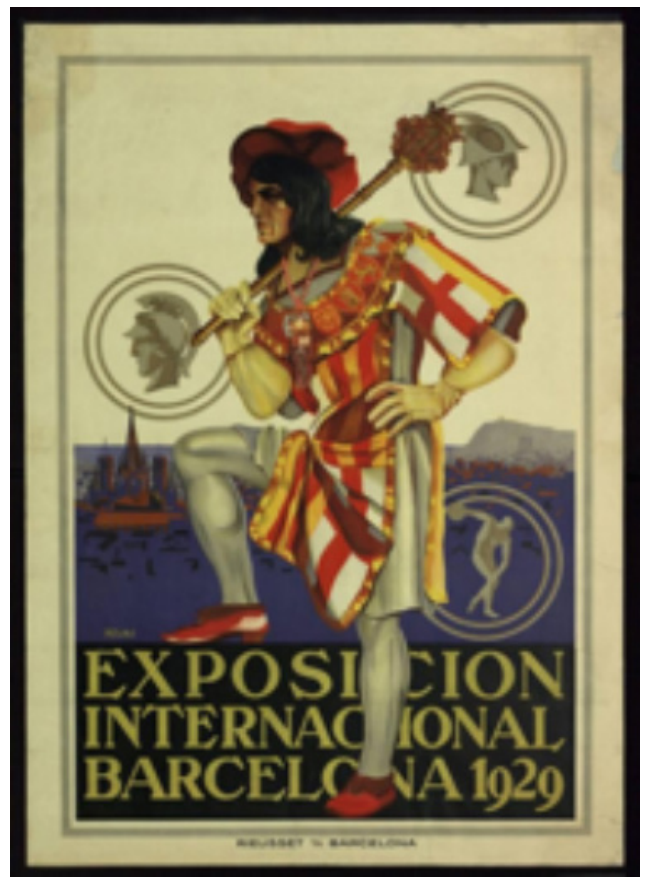

Imagen 3. Cartel de la exposición de Barcelona del dibujante y pintor Josep Rojas Assens. Esta litografía ganó el primer premio del concurso de la Exposición Internacional de Barcelona de 1929. La obra representa a un macero del Ayuntamiento de Barcelona sobre el perfil de la ciudad ${ }^{20}$.

19. Sin firmar. (Junio de 1930). Orfeó Gracienc-Cobla Barcelona. Música, n. ${ }^{\circ}$ 10, 18.

20. Localizado en la Biblioteca de Cataluña con la signatura: Cartells II 43. También se puede consultar en la Memoria Digital de Cataluña. Disponible en: $<$ http://mdc.csuc.cat $/ \mathrm{cdm} /$ singleitem/collection/cartellsBC/id/151/rec/1> [Última consulta: 01/07/2019]. 
PAULA MOLINA GONZÁLEZ Y REBECA GEA MARTÍNEZ

UN VIAJE TURÍSTICO-MUSICAL POR ESPAÑA EN 1930.

APROXIMACIÓN A LOS ACTOS DE BARCELONA Y VALENCIA

Cabe mencionar que, además de la programación que finalmente se llevó a cabo, Kurt Schindler había considerado la posibilidad de añadir al programa dos conciertos más de instrumentos solistas. Uno de ellos habría sido de piano, en el cual se habría prestado un especial interés por la música de los compositores catalanes, como Enrique Granados o Isaac Albéniz, ambos representantes del nacionalismo musical español. En la correspondencia de Schindler a Pujol, se especifica esta cuestión dando más importancia al repertorio que se pretendía difundir que al intérprete que lo ejecutaría, ya que no se hace referencia a ningún pianista en concreto. En contraposición, en el otro concierto propuesto, se especificaba que el intérprete fuese el guitarrista Miguel Llobet ${ }^{21}$. En este caso, no hay referencias al repertorio que debía interpretar, sin embargo, podemos suponer que el interés estaba en que los turistas asistiesen a un concierto de guitarra española, llevado a cabo por uno de los guitarristas más destacados de la época. Además, Miguel Llobet era catalán y había trabajado la música popular de su región, habiendo armonizado y arreglado diversas canciones populares catalanas para guitarra, las cuales podría haber interpretado en dicho concierto. El hecho de haber realizado estos conciertos habría supuesto cierta variedad en el programa del viaje, ya que exceptuando el realizado en Sevilla por el primer organista de la Catedral de dicha ciudad, Norberto Almandoz, no se dio espacio a intérpretes solistas, sino que todos los conciertos fueron de agrupaciones musicales.

\section{VALENCIA}

Los eventos festivo-musicales del viaje en Valencia se realizaron durante los días 19 y 20 de mayo de 1930, siendo esta la segunda ciudad del programa ya citado. La organización y la supervisión musical de dichos eventos estuvieron a cargo del distinguido músico valenciano Eduardo López-Chávarri.

Como se detalla en las fuentes hemerográficas anteriores, los viajeros llegaron a Valencia el día 19 por la tarde y durante el día 20 disfrutaron de una fiesta en los Jardines del Real, también conocidos como Jardines de Viveros. Este acto constó de dos eventos. Por un lado, un concierto de música valenciana a cargo de la Banda Municipal de Valencia, bajo la dirección de Luis Ayllonal. Por otro lado, se ejecutaron bailes regionales valencianos acompañados por una rondalla y una cantadora, coordinados por Enrique Vicent. En la noticia del 4 de mayo de 1930 del periódico $A B C$, donde también se detalla el programa musical del viaje en relación a Valencia, solo se hace referencia a sus bailes regionales, detallando que estos se ejecutarán con «trajes valencianos y acompañamiento de una orquesta

21. Schindler, K. (3 de abril de 1930). [Carta para Francesc Pujol]. Biblioteca del Orfeó Català. Fondo Francesc Pujol. Signatura: CAT CEDOC 3.2 2367-2377. 
PAULA MOLINA GONZÁLEZ Y REBECA GEA MARTÍNEZ

UN VIAJE TURÍSTICO-MUSICAL POR ESPAÑA EN 1930.

APROXIMACIÓN A LOS ACTOS DE BARCELONA Y VALENCIA

típica» ${ }^{22}$. Entendemos que por orquesta típica se refiere a aquello descrito en el periódico La Época, es decir, una «rondalla» que acompañaba a una voz femenina.

Sin embargo, a través de la correspondencia mantenida entre Kurt Schindler y Eduardo López-Chávarri, se observan algunos detalles de la organización previa a la fiesta en Valencia, los cuales difieren ligeramente del resultado final ya citado ${ }^{23}$. Ambos estuvieron en contacto desde el 11 de marzo de 1930 (la primera carta de Schindler a López-Chávarri) hasta abril. Teniendo en cuenta que la tercera carta de Schindler a López-Chávarri fue el 9 de abril, la repuesta de López-Chávarri se efectuaría unos días después de esta fecha ${ }^{24}$.

Según el programa ya citado, los eventos musicales en Valencia se basaron en un concierto de la Banda Municipal y en la exhibición de bailes populares valencianos. Aunque el plan inicial no fue este, Eduardo López-Chávarri sugirió utilizar una orquesta como medio de expresión de la música valenciana de ese momento, en vez de una banda. Kurt Schindler estuvo de acuerdo, tal y como explica en una de las cartas a López-Chávarri, ya que Kurt Schindler manifiesta que «está incondicionalmente de su parte [de López-Chávarri] en esta cuestión» ${ }^{25}$.

No obstante, debido al cambio de gobierno que se produjo durante las fechas previas al viaje, esto es, la dictadura de Dámaso Berenguer la cual se formó en enero de 1930, la idea de López-Chávarri no se pudo llevar a cabo, ya que el Patronato de Turismo no pudo encargarse de la gestión económica de la Orquesta de Valencia durante los días de la fiesta ${ }^{26}$. Por esta razón, Schindler le sugirió a López-Chávarri que propusiera una alternativa, por ejemplo, que la Sociedad Filarmónica de Valencia buscara alguna otra agrupación orquestal más pequeña para un concierto, coincidiendo con la estancia de los viajeros en Valencia.

Además, estas fuentes documentales nos ofrecen datos sustanciales para la reconstrucción de dicho evento, sobre todo relacionados con las personas que intervinieron en la materialización de la fiesta en Valencia. Asimismo, nos da una ligera perspectiva del trato personal que pudo existir entre Schindler y López-Chávarri.

22. Sin firmar. (4 de mayo de 1930). $A B C$ (Madrid), p. 38.

23. En nuestro caso, solo hemos tenido acceso a las cartas de Kurt Schindler a Eduardo López-Chávarri, facilitadas amablemente por la Biblioteca Valenciana. Véase el apartado Epistolario para conocer la referencia exacta de dicha correspondencia. Desafortunadamente, no hemos tenido acceso a las respuestas de Eduardo López-Chávarri a Kurt Schindler. Sospechamos que pueden encontrarse en el mismo archivo personal de López-Chávarri, depositado en la ya citada biblioteca, aunque no hemos podido localizarlas aún.

24. Véase el apartado Epistolario.

25. Schindler, K. (9 de abril de 1930). [Carta para Eduardo López-Chávarri]. Biblioteca Valenciana Nicolau Primitiu. Fondo Familia Eduardo López-Chávarri Andújar. Signatura: AELCHE CR 2531.

26. Schindler, K. Ibíd. 
PAULA MOLINA GONZÁLEZ Y REBECA GEA MARTÍNEZ

UN VIAJE TURÍSTICO-MUSICAL POR ESPAÑA EN 1930.

APROXIMACIÓN A LOS ACTOS DE BARCELONA Y VALENCIA

Como comentábamos anteriormente, José Antonio de Sangróniz y Castro, como delegado del Patronato de Turismo, fue el enlace principal para que la idea del viaje se materializara. No obstante, parece ser, según la correspondencia entre Schindler y López-Chávarri, que Sangróniz se sentía especialmente interesado por la fiesta en Valencia, ya que, nada más hablar con Schindler en enero, se reúne en Valencia con varias personalidades artísticas y políticas para fijar algunos aspectos de la fiesta. Incluso se reunió con el entonces subdelegado de Turismo de Valencia, Enrique Carlos de Castellví y Hortega de Medina, conde de la Villanueva, barón de Torres-Torres y marqués de Laconi, el cual se mostró muy entusiasmado con los festejos valencianos para el viaje.

Finalmente, la correspondencia entre ambos detalla que Kurt Schindler visitó Valencia unos años antes a 1930 y que conoció por aquel entonces a Eduardo López-Chávarri, tal como se observa en la siguiente frase del propio Schindler: "Creo no tener necesidad de recordarle quien soy, ya que seguramente no me habrá olvidado desde mis dos visitas en años pasados a esa hermosa capital» ${ }^{27}$. Podemos suponer que ambos podrían haber mantenido algo más que una relación formal y cordial, llegando a establecer una amistad.

\section{CONCLUSIONES}

Se observa a través de este artículo una faceta poca conocida del músico alemán Kurt Schindler, tal y como cita Matilde Olarte (2009: 95), la cual fue:

Su interés por mostrar la cultura española (música, tradiciones y costumbres) a sus múltiples discípulas y admiradoras neoyorkinas en los años previos a nuestra guerra civil y que nos responde a muchos de los interrogantes del porqué de su afición a nuestro país.

Aunque el anuncio se imprimió en francés, alemán e inglés, según lo señalado por Kurt Schindler a Eduardo López-Chávarri ${ }^{28}$, sí que es cierto que la proporción de folletos en inglés fue mayor que aquellos impresos en alemán y en francés, con lo cual según la relación económica y hasta cierto modo empresarial de Schindler

27. Schindler, K. (11 de marzo de 1930). [Carta para Eduardo López-Chávarri]. Biblioteca Valenciana Nicolau Primitiu. Fondo Familia Eduardo López-Chávarri Andújar. Signatura: AELCHE CR 2529.

28. En la carta del día 9 de abril, se dice que se imprimirán del anuncio del viaje turístico-musical «2.500 folletines Ingleses, 2.500 folletines Españoles, 1.500 folletines Alemanes y 1.500 folletines Franceses», en: Schindler, K. (9 de abril de 1930). [Carta para Eduardo López-Chávarri]. Biblioteca Valenciana Nicolau Primitiu. Fondo Familia Eduardo López-Chávarri Andújar. Signatura: AELCHE CR 2531. 
PAULA MOLINA GONZÁLEZ Y REBECA GEA MARTÍNEZ

UN VIAJE TURÍSTICO-MUSICAL POR ESPAÑA EN 1930.

APROXIMACIÓN A LOS ACTOS DE BARCELONA Y VALENCIA

con Archer Milton Huntington, se hace evidente que se buscaba atraer mayoritariamente a público norteamericano.

Por un lado, antes cabe destacar que sería interesante contrastar los aspectos tratados en las fuentes hemerográficas ya citadas con los anuncios en las otras lenguas. De esta forma, se podría saber si el acercamiento fue el mismo para el público español que para el extranjero.

Por otro lado, queremos resaltar una incógnita que nos surge al no haber encontrado hasta el momento fuentes que aporten información al respecto, ya que no sabemos cómo se realizó la elección de los lugares que se visitaron en el viaje y, sobre todo, por qué se descartaron otros sitios como Galicia, en los que también había un rico folklore musical.

Finalmente, podemos decir que las fuentes encontradas y mencionadas en el presente artículo suponen un material de gran utilidad para el estudio del contexto de la primera mitad del siglo XX, momento en el cual se empezó a mostrar interés por la recopilación del folklore de nuestro país. Aunque nos hayamos centrado en un asunto en particular, es decir, el viaje turístico-musical realizado por España, este material documental es el reflejo de las redes que se generaron y, por tanto, nos aporta una información fundamental para conocer a figuras como el musicólogo Kurt Schindler y de qué manera estuvieron implicadas en este proceso de compilación y difusión de la música tradicional española.

\section{FUENTES HEMEROGRÁFICAS}

Sin firmar. (Abril 1930). Viaje turístico musical por España. Revista de las Españas, 44.

Sin firmar. (4 de mayo de 1930). $A B C$ (Madrid), 38.

Sin firmar. (8 de mayo de 1930). Una bella iniciativa de arte. Excusión turístico-musical a través de España. La Libertad (Madrid), 8.

Sin firmar. (13 de mayo de 1930). Excursión turístico-musical por España. La Época, n. ${ }^{\circ} 28,1$. Sin firmar. (14 de mayo de 1930). Palacio de Proyecciones. La Vanguardia, 22.

Sin firmar. (Junio de 1930). Orfeó Català. Música, n. ${ }^{\circ} 10,20$.

Sin firmar. (Junio de 1930). Orfeó Gracienc-Cobla Barcelona. Música, n. ${ }^{\circ} 10,18$.

Amiguet, T. (2015). 1929: la Exposición que internacionalizó Barcelona. La Vanguardia.

Disponible en: <https://www.lavanguardia.com/hemeroteca/20150115/54423264283/ exposicion-internacional-de-1929-barcelona-1930-ferias-urbanismo-arquitectura. html> [Última consulta: 05/05/2019].

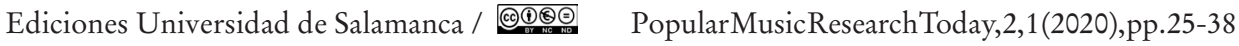


PAULA MOLINA GONZÁLEZ Y REBECA GEA MARTÍNEZ

UN VIAJE TURÍSTICO-MUSICAL POR ESPAÑA EN 1930.

APROXIMACIÓN A LOS ACTOS DE BARCELONA Y VALENCIA

\section{EPISTOLARIO}

Schindler, K. (11 de marzo de 1930). [Carta para Eduardo López-Chávarri]. Biblioteca Valenciana Nicolau Primitiu. Fondo Familia Eduardo López-Chávarri Andújar. Signatura: AELCHE CR 2529.

Schindler, K. (3 de abril de 1930). [Carta para Eduardo López-Chávarri]. Biblioteca Valenciana Nicolau Primitiu. Fondo Familia Eduardo López-Chávarri Andújar. Signatura: AELCHE CR 2530.

Schindler, K. (9 de abril de 1930). [Carta para Eduardo López-Chávarri]. Biblioteca Valenciana Nicolau Primitiu. Fondo Familia Eduardo López-Chávarri Andújar. Signatura: AELCHE CR 2531.

Schindler, K. (3 de abril de 1930). [Carta para Francesc Pujol]. Biblioteca del Orfeó Català. Fondo Francesc Pujol. Signatura: CAT CEDOC 3.2 2367-2377.

\section{BIBLIOGRAFÍA}

Katz, Israel. J. (2001). López-Chávarri Marco, Eduardo. Oxford Music Online. Disponible en: <https://www.oxfordmusiconline.com/search?q=chavarri\&searchBtn=Search\&isQuickSearch=true $>$ [Última consulta: 26/05/2019].

Katz, Israel. J. (2001). Schindler, Kurt. Oxford Music Online. Disponible en: <https://www. oxfordmusiconline.com/grovemusic/view/10.1093/gmo/9781561592630.001.0001/ omo-9781561592630-e-0000024872> [Última consulta: 30/05/2019].

Martín Emparan, A. (2008). El diseño gráfico en la Exposición Ibero Americana de Sevilla 1929 [Tesis doctoral]. Málaga: Universidad de Málaga.

Olarte Martínez, M. (2009). Apuntes de Sevilla a través de intrépidas jovencitas americanas de los años 30: Bienvenido Mr. Schindler. En M. A. Berlanga Fernández, Lo Andaluz Popular, Símbolo de lo Nacional (pp. 95-111). Granada: Editorial de la Universidad de Granada.

Sapena Martínez, S. (2007). La sociedad filarmónica de Valencia (1911-1945): Origen y consolidación [Tesis doctoral]. Valencia: Universidad Politécnica de Valencia.

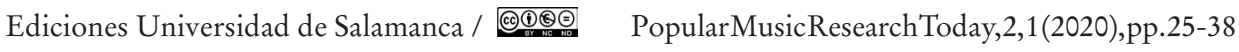

